007

\section{ANALYSIS OF THE LEFT VENTRICULAR ASSIST DEVICE (LVAD) ACOUSTIC SIGNAL: A NOVEL CLINICAL DIAGNOSTIC METHOD FOR SUSPECTED PUMP THROMBOSIS}

M R Noor, ${ }^{1}$ N Hitchins, ${ }^{2}$ E Doyle, ${ }^{2}$ N R Banner, ${ }^{1} \mathrm{~K}$ Parker, ${ }^{2} \mathrm{C}$ Bowles ${ }^{1}{ }^{1}$ Royal

Brompton and Harefield NHS Foundation Trust; ${ }^{2}$ Imperial College London

doi:10.1136/heartjnl-2013-304019.7

Introduction Left Ventricular Assist Devices (LVADs) are an established treatment for advanced heart failure in appropriately selected patients. Pumps have gradually become smaller and more durable, are associated with survival benefits, improvement in functional status and quality of life in patients with advanced heart failure. Despite impressive advances in device technology, pump thrombosis remains a serious complication and a definitive diagnostic method is currently unavailable. Rotary pumps produce a characteristic acoustic signal which is primarily determined by rotation speed and impeller symmetry. Our group have evaluated a novel method of pump thrombus diagnosis based on the analysis of the acoustic signal produced by implanted LVADs. The aim of this study was to compare the acoustic characteristics of normal devices with those with suspected pump thrombosis.

Methods The control group consisted of 20 ambulatory heart failure patients receiving support with an axial impeller Thoratec HeartMate II $(n=10)$ or centrifugal HeartWare HVAD $(n=10)$. The pump thrombosis group consisted of four patients, that is, Heartmate II $(n=2)$, HVAD $(n=2)$. Recordings were made with the patient in a semi-recumbent position at the clinical pump speed (typically circa $9000 \mathrm{rpm}$ for HeartMate II and $3000 \mathrm{rpm}$ for HVAD) with purpose made recording stethoscope placed on the chest wall in the 'mitral' position and were analysed using analysis software developed in house (Matlab, Mathworks Inc). Fast Fourier Transformation (FFT) of the acoustic signal was used in order to reveal the constituent frequency components.

Results Acoustic profiles were readily obtainable from all patients and the FFT spectra were dependent on the LVAD impeller symmetry and rotation speed. In HeartMate II (HMII) patients, FFT spectral peaks were present at the impeller rotation frequency $152.1 \pm 2.5 \mathrm{~Hz}$ and blade passing frequency of 3 times this value $(456.1 \pm 7.4 \mathrm{~Hz}$ ), reflecting the presence of 3 flow channels in the impeller. For HVAD recipients, FFT spectral peaks were present at the impeller rotation frequency $48.6 \pm 2.2 \mathrm{~Hz}$ and blade passing frequency $194.5 \pm 8.6 \mathrm{~Hz}$, reflecting the presence of four impeller flow channels. In LVAD patients with suspected pump thrombosis, additional FFT spectral peaks to those described above were detected. Successful resolution of the pump thrombus episodes (normalisation of pump power and blood trauma biomarkers) in the two HVAD patients after 6 and 14 days was associated with normalisation of the FFT traces.

Conclusions The appearance of atypical FFT spectral peaks during pump thrombus episodes is likely to be related to a combination of unbalanced impeller motion, changes in blood flow and increased turbulence. Acoustic monitoring is a simple non-invasive method which is likely to have clinical utility in the diagnosis of pump thrombus episodes in LVAD patients. 